\title{
Thoracic paravertebral regional anesthesia improves analgesia after breast cancer surgery: a randomized controlled multicentre clinical trial
}

\section{L'anesthésie régionale paravertébrale thoracique améliore l'analgésie après chirurgie pour cancer du sein: essai clinique multicentrique randomisé contrôlé}

\author{
Jiang Wu, MD • Donal Buggy, MD • Edith Fleischmann, MD • \\ Ivan Parra-Sanchez, MD • Tanja Treschan, MD • Andrea Kurz, MD • \\ Edward J. Mascha, PhD • Daniel I. Sessler, MD \\ Received: 24 April 2014/Accepted: 24 November 2014/Published online: 6 December 2014 \\ (C) Canadian Anesthesiologists' Society 2014
}

\begin{abstract}
Background The contribution of regional anesthesia with thoracic paravertebral blockade to postoperative analgesia remains unclear. We compared the effect of a combination of paravertebral blockade and propofol general anesthesia (GA) with sevoflurane GA and opioid analgesia on postoperative pain and opioid use for patients undergoing breast cancer surgery.

Methods Patients having breast cancer surgery were randomly assigned to paravertebral analgesia with propofol GA (PPA, $n=187)$ or sevoflurane GA with

Author contributions Jiang Wu, Donald J. Buggy, Edith Fleischmann, Ivan Parra-Sanchez, Tanja Treschan, and Andrea Kurz contributed substantially to the acquisition of data. Jiang $W u$, Donald J. Buggy, Edith Fleischmann, Ivan Parra-Sanchez, Tanja Treschan, Andrea Kurz, Edward J. Mascha, and Daniel I. Sessler contributed substantially to the interpretation of data. Donald J. Buggy, Edith Fleischmann, Ivan Parra-Sanchez, Tanja Treschan, Andrea Kurz, and Edward J. Mascha contributed to the analysis of data. Donald J. Buggy and Daniel I. Sessler contributed substantially to the conception and design of the manuscript. Donald J. Buggy, Jiang Wu, and Daniel I. Sessler drafted the article.
\end{abstract}

J. Wu, MD · I. Parra-Sanchez, MD

Anesthesiology Institute, Cleveland Clinic, Cleveland, OH, USA

D. Buggy, MD ( $\square)$

Department of Anaesthesia, Mater Misericordiae University Hospital, National Cancer Screening Service \& University College Dublin, Dublin 7, Ireland

e-mail: donal.buggy@ucd.ie

\section{E. Fleischmann, MD}

Department of Anesthesiology and Intensive Care, Medical University Vienna, Vienna, Austria perioperative opioid analgesia (SOA, $n=199$ ). The PPA and SOA groups were compared for opioid consumption and pain outcomes (on a 0-10 visual analogue scale [VAS]) at two hours postoperatively using superiority and inferiority statistics. We compared our results with previous publications in a meta-analysis.

Results Compared with the SOA group, the PPA group experienced reduced median [interquartile range] pain VAS scores (1 [1,3] vs 2.5 [1,4], respectively; median difference -1.0; $99 \%$ confidence intervals [CI]: -1.5 to $-0.5)$ and required less intraoperative fentanyl (50 [0, 125] $\mu \mathrm{g}$ vs 200 [100, 300] $\mu \mathrm{g}$, respectively; median difference -100; 99\% CI: -150 to -100) and less longacting opioid $(0[0,0] \mathrm{mg}$ vs $3.0[0,12] \mathrm{mg}$, respectively, morphine equivalents; median difference -3 ; $99 \%$ CI: -4 to -2). Thus, non-inferiority was detected for all the above outcomes, and superiority tests for each outcome were highly significant in the expected directions $(P<0.001)$. Meta-analysis, including the current study, estimated a reduction in worst pain of 2.3 points (95\% CI: 1.8 to 2.8 ) on a $0-10$ scale and a $72 \%$ reduction (95\% CI: 42 to 87 ) in

\section{T. Treschan, MD}

Department of Anesthesiology, Düsseldorf University Hospital, Düsseldorf, Germany

\section{A. Kurz, MD · D. I. Sessler, MD}

Department of Outcomes Research, Cleveland Clinic, Cleveland, $\mathrm{OH}$, USA

\section{E. J. Mascha, $\mathrm{PhD}$}

Departments of Quantitative Health Sciences and Outcomes Research, Cleveland Clinic, Cleveland, OH, USA 
mean opioid consumption in the immediate two postoperative hours for PPA vs SOA.

Conclusion Our results were largely consistent with previous much smaller studies. Compared with sevoflurane GA with opioid analgesia, the combination of paravertebral analgesia with propofol GA provides an early clinical analgesic benefit in females having breast cancer surgery. This analysis is a substudy of an ongoing multicentre double-blinded randomized trial (www. clinicaltrials.gov, NCT00418457) of cancer recurrence.

\section{Résumé}

Contexte La contribution de l'anesthésie régionale avec bloc paravertébral thoracique à l'analgésie postopératoire reste mal connue. Nous avons comparé l'effet de la combinaison bloc paravertébral et anesthésie générale (AG) au propofol à l'effet de l'AG au sévoflurane et analgésie par opiö̈des sur le niveau de douleur postopératoire et l'utilisation des opiö̈des chez des patientes subissant une chirurgie pour cancer du sein.

Méthodes Des patientes devant subir une chirurgie pour cancer du sein ont été randomisées dans deux groupes pour recevoir une AG par propofol et analgésie paravertébrale (PPA, $n=187$ ) ou une AG par sévoflurane avec analgésie périopératoire par opiö̈des (SOA, $n=199)$. Les groupes PPA et SOA ont été comparés sur le plan de la consommation des opiö̈des et des niveaux de douleur (sur une échelle visuelle analogique [EVA] de 0 à 10) à deux heures postopératoires utilisant des calculs statistiques de supériorité et d'infériorité. Nous avons comparé nos résultats avec les publications précédentes dans une méta-analyse.

Résultats Comparé au groupe SOA, le groupe PPA a présenté des scores médians (intervalle interquartile) de douleur réduits sur l'EVA (respectivement, 1 [1,3] contre 2,5 [1,4]; différence des médianes -1,0; intervalle de confiance [IC] à $99 \%:-1,5$ à-0,5) et a nécessité moins de fentanyl peropératoire (respectivement, 50 [0 à 125] $\mu \mathrm{g}$ contre 200 [100 à 300] $\mu \mathrm{g}$; différence des médianes -100; IC à $99 \%$ : -150 à -100) et moins d'opioüdes à longue durée d'action (respectivement, 0 [0,0] mg contre 3,0 [0 à 12] mg d'équivalent-morphine; différence des médianes -3; IC à $99 \%$ : -4 à-2). Ainsi, une non-infériorité a été détectée pour tous les critères d'évaluation ci-dessus et les tests de supériorité pour chaque critère ont été hautement significatifs dans le sens attendu $(P<0,001) . \quad L a$ méta-analyse incluant l'étude actuelle a estimé une réduction de la pire douleur de 2,3 points (IC à $95 \%$ : 1,8 à 2,8) sur une échelle de 0 à 10 et une diminution de $72 \%$ (IC à $95 \%: 42$ à 87) de la consommation moyenne d'opiödes dans les deux heures postopératoires immédiates pour le groupe PPA par rapport au groupe SOA.
Conclusion Nos résultats ont été largement concordants avec des études antérieures beaucoup plus petites. Comparativement à l'AG au sévoflurane avec analgésie par opiö̈des, l'association d'une analgésie paravertébrale et d'une AG au propofol a fourni des avantages cliniques précoces pour les femmes subissant une chirurgie pour cancer du sein. Cette analyse est une sous-étude d'un essai randomisé multicentrique à double insu sur la récidive cancéreuse (www.ClinicalTrials.gov: $n^{\circ}$ NCT00418457).

Thoracic paravertebral analgesia appears to be a useful adjunct for breast surgery. It is hardly surprising that the technique provides excellent analgesia and therefore reduces the need for deep general anesthesia (GA). ${ }^{1-4}$ Nevertheless, the extent to which paravertebral analgesia reduces the requirement for intraoperative volatile anesthetics and postoperative opioid analgesia remains unclear, with current estimates based on single-centre reports with a small sample size.

The extent to which paravertebral analgesia reduces the need for volatile anesthesia and opioids is potentially important because volatile anesthesia has been shown to impair numerous immune functions, including those of neutrophils, macrophages, dendritic cells, T-cells, and natural killer cells. ${ }^{5}$ In contrast, propofol is variously reported to be inhibitory, ${ }^{6}$ have little effect, ${ }^{7}$ or even enhance immune function. ${ }^{8}$ Opioid analgesics inhibit both cellular and humoral immune function in humans. ${ }^{9,10}$ Furthermore, morphine is pro-angiogenic and promotes breast tumour growth in rodents. ${ }^{11}$ Consistent with this theory, surgical studies in animals indicate that regional anesthesia and optimum postoperative analgesia independently reduce the metastatic burden in animals inoculated with breast adenocarcinoma cells. ${ }^{12}$ Furthermore, a small retrospective analysis in cancer patients suggests that paravertebral analgesia reduces the risk of recurrence. Nevertheless, retrospective clinical studies cannot prove a cause-and-effect link. ${ }^{1,13}$

This report is an interim analysis of a trial designed ${ }^{13}$ with cancer recurrence and chronic post-surgical pain as the primary and secondary outcomes, respectively. Available data to date consist of anesthetic technique endpoints and analgesia/pain outcomes. Therefore, in this present report, we tested the hypothesis that a combination of paravertebral analgesia with propofol anesthesia (PPA) would be non-inferior to a combination of sevoflurane GA with perioperative opioid analgesia (SOA) on postoperative pain score outcomes, the need for intraoperative and postoperative opioids, and the use of propofol and sevoflurane. Furthermore, we hypothesized that the PPA 
technique would be superior to the SOA technique in at least one of these same endpoints and outcomes.

We also performed a meta-analysis to compare our results with previous studies.

\section{Methods}

This analysis is a substudy of an ongoing multicentre double-blinded randomized trial of cancer recurrence; the full protocol has previously been published. ${ }^{13}$ All patients were enrolled after Institutional Review Board approval and informed consent. No cancer outcomes from the primary trial were evaluated; the purpose of this analysis was simply to confirm that the randomized groups differed substantially on key mechanistic factors. This study was approved by the Research Ethics Committees or Institutional Review Boards for Ethics in Research of all participating centres (Appendix).

Briefly, we enrolled women scheduled for full mastectomy or partial mastectomy with node dissection because of stage 1-3 breast cancer. They were randomly assigned to thoracic paravertebral analgesia with propofol GA (PPA group) or to sevoflurane GA combined with perioperative opioid analgesia (SOA group) using computer-generated codes that were stratified by site. Allocations were assigned through a web-based system that was accessed shortly before induction of anesthesia.

\section{Protocol}

In patients assigned to the SOA group, anesthesia was induced with fentanyl $1-3 \mu \mathrm{g} \cdot \mathrm{kg}^{-1}$ and propofol $2-4 \mathrm{mg} \cdot \mathrm{kg}^{-1}$ and maintained with sevoflurane in $80 \%$ oxygen, balance nitrogen, and fentanyl. Sevoflurane and fentanyl administration were adjusted to maintain blood pressure and heart rate within $20 \%$ of preoperative values. Intravenous morphine sulphate was titrated to a respiratory rate of 12-14 breaths $\min ^{-1}$ near the end of surgery. Postoperative analgesia was morphine or a similar longacting opioid, provided as needed either intravenously or via patient-controlled pump. After approximately $24 \mathrm{hr}$, the patients were transitioned to acetaminophen and nonsteroidal anti-inflammatory analgesics.

In patients assigned to the PPA Group, analgesia was largely provided by paravertebral blockade. ${ }^{14}$ Paravertebral anesthesia was provided either with a thoracic (T) interspace 2-4 catheter or multilevel injections from $\mathrm{T} 1$ to $\mathrm{T} 5 .{ }^{15}$ When a catheter was used, it was inserted into the ipsilateral paravertebral space at the level of $\mathrm{T} 2 / 3$ or T3/4 using a standard technique. ${ }^{2}$ Patients were given a bolus of $0.5 \%$ bupivacaine $10-20 \mathrm{~mL}$ or $0.5 \%$ ropivacaine with epinephrine after a test dose with $1.5 \%$ lidocaine and
1:200,000 epinephrine. Near the end of surgery, an infusion of either solution was started at a rate of $6-10 \mathrm{~mL} \cdot \mathrm{hr}^{-1}$, and the infusion rate was reduced or increased as deemed necessary by the attending anesthesiologist. The local anesthetic infusion was continued as clinically necessary up to $48 \mathrm{hr}$. When a multilevel technique was used, $0.75 \%$ ropivacaine $5 \mathrm{~mL}$ was given at each of the five levels. Additionally, injections of $0.5 \%$ ropivacaine were given by the surgeon to block cervical and contralateral thoracic nerves that also contribute to the innervation of the breast.

In the PPA group, paravertebral anesthesia was supplemented with deep propofol sedation/GA (usually infused at a rate of $60-90 \mu \mathrm{g} \cdot \mathrm{kg}^{-1} \cdot \mathrm{min}^{-1} i \mathrm{v}$ ), with larger amounts being given per discretion of the attending anesthesiologist depending on the adequacy of the block, the extent and duration of surgery, and patient cooperation. This is the strategy used in nearly all previous studies of paravertebral analgesia for breast surgery. ${ }^{3}$ A laryngeal mask was inserted if necessary in patients receiving larger propofol doses. The addition of sevoflurane and/or fentanyl was permitted if insertion of an endotracheal tube was required due to the need for deeper anesthesia. Postoperatively, analgesia was provided primarily by the regional block and supplemented with acetaminophen and/ or nonsteroidal anti-inflammatory drugs. Supplemental morphine or a similar long-acting opioid was provided if pain relief was inadequate. Patients were transitioned to acetaminophen and/or nonsteroidal analgesics at about 24 hr. Clinicians were not blinded to anesthetic technique.

\section{Measurements}

The volatile anesthetic dose was measured in MAC hours, and intraoperative use of fentanyl was recorded. The use of intraoperative and long-acting opioids in the immediate two postoperative hours was also recorded. Long-acting opioids were converted to morphine sulphate equivalents using conventionally suggested ratios. ${ }^{16}$ Piritramid is commonly used in Europe and was converted at a ratio of $15 \mathrm{mg}$ piritramid to $1 \mathrm{mg}$ of morphine sulphate. ${ }^{17,18}$ To provide pain assessment, worst surgical site pain in the immediate two postoperative hours was recorded on $0-10$ point visual analogue scale (VAS). ${ }^{19}$

Statistical analysis

Analysis included all patients randomized from November 2008 to October 2010. To test our hypotheses in comparing the PPA group with the SOA group, our primary outcomes and endpoints were pain (reduction) at two hours postoperatively, the use of intraoperative and postoperative long-acting opioids (reduction), use of sevoflurane (reduction), and use of propofol (increase). 
Our primary analysis followed intent-to-treat principles. For missing data, we implemented intent-to-treat by conservatively assigning the worst observed outcome to PPA patients and the best observed outcome to SOA patients. In a secondary analysis, we used available data analysis that included only non-missing primary outcome data for the five primary outcomes.

We planned a priori to claim PPA more effective than SOA only if it could be shown to be non-inferior to SOA on all primary outcomes and endpoints (referred to collectively as "outcomes" in the remainder of this Methods section) and superior to SOA on at least one. $^{20,21}$ Our joint hypothesis testing framework across these outcomes thus consisted of the following null and alternative hypotheses for PPA $v s$ SOA.

H01: $P P A$ inferior to $S O A$ on $\geq 1$ outcome or H02: PPA superior to $S O A$ on none.

HA1: $P P A$ non-inferior to $S O A$ on all and HA2: $P P A$ superior to $S O A$ on $\geq 1$ outcome.

Since both non-inferiority on all outcomes (HA1) and superiority on at least one outcome (HA2) were required to claim PPA better than SOA, this was considered an intersection-union test. ${ }^{22}$ Therefore, no adjustment to the significance criterion for testing both non-inferiority and superiority was required, and both were tested at the chosen alpha level of 0.025 .

Non-inferiority was tested at the 0.025 level for each outcome in one-tailed tests. Since non-inferiority was required on all outcomes, the non-inferiority testing itself was also an intersection-union test, so that no adjustment to the significance criterion for multiple non-inferiority tests was required. If non-inferiority was claimed on all outcomes, superiority would be tested on each outcome with one-tailed tests (Wilcoxon rank-sum test) in the hypothesized direction (i.e., reduction or increase) at the overall 0.025 level, adjusting for multiple comparisons with the Bonferroni correction $(0.005$ one-tailed significance criterion).

We a priori defined the non-inferiority delta for pain as one point on the VAS. We used the difference between medians as the main outcome measure for pain score and reported it only for descriptive purposes for the other variables. The non-inferiority delta for intraoperative fentanyl consumption and long-acting opioid consumption was a priori defined as a ratio of mean ranks of 1.1, since lower values for these parameters were desirable.

We used a ratio of mean ranks instead of ratio of medians or means because these variables were not normally or even log-normally distributed. Furthermore, the distributions did not have similar shapes between the two groups, making a comparison of medians inappropriate and potentially disjoint from the Wilcoxon rank-sum test results. For example: a ratio of mean ranks of 1.1 indicates that, when an outcome variable is ranked from smallest to largest across all patients, the average ranking (or equivalently, the average percentile) for the PPA group is $10 \%$ higher than the average ranking for the SOA group or that PPA increased the mean ranking by $10 \%$. Likewise, a ratio of 0.5 would indicate that patients in the PPA group had a $50 \%$ reduction in the mean ranking for that outcome.

The percentile bootstrap resampling method was used to estimate the $99 \%$ confidence interval (CI) for the difference in medians and ratio of mean ranks for all outcome measures comparing the PPA and SOA groups. ${ }^{23}$ Noninferiority was claimed if the upper confidence limit was below the non-inferiority delta (except for propofol, for which the lower confidence limit needed to be above the non-inferiority delta).

To have $90 \%$ power at the 0.025 significance level to detect superiority on at least one of the four outcomes with a one-tailed test, a total sample size of 50 per group would be needed. This a priori assumes variability equal to what was observed in the current dataset and differences of $20 \%$ of the control mean for each of opioid consumption, sevoflurane, and propofol, plus a difference of 2 for VAS pain, and adjusts for multiple comparisons.

Results are presented as mean (SD), median [interquartile range], or median difference ratio of mean ranks $(99 \% \mathrm{CI})$. $\mathrm{SAS}^{\circledR}$ statistical software 9.4 (SAS Institute; Cary, NC, USA) was used for all analyses.

\section{Meta-analysis}

We conducted a meta-analysis to assess the effect of paravertebral anesthesia alone (or the combination of paravertebral blockade and GA) vs GA alone on each of worst pain score and cumulative analgesic use in the immediate postoperative two hours. The systematic search, data extraction, critical appraisal, and pooled analysis were performed according to the PRISMA statement. We used the same search criteria as in a similar previous metaanalysis ${ }^{4}$ and similarly restricted our analysis to randomized trials of breast surgery. Briefly, we retrieved all randomized trials comparing paravertebral blocks (with or without concomitant GA) with GA alone. Two primary outcomes of interest were defined in our secondary screen: 1) worst pain score in the immediate two postoperative hours and 2) actual opioid consumption in the immediate two postoperative hours.

In our meta-analysis, different analgesic units across studies were converted to morphine units using standard formulae. Log-normal distribution was assumed for morphine consumption, so that the treatment effect of PPA vs SOA was expressed as the ratio of geometric means. The treatment effect on mean worst pain score in 
the two hours post-surgery was expressed as the difference in means on a 0-10 scale (adopted as necessary from a VAS score). Random effects models were used for both outcomes to be conservative.

Heterogeneity of the effect sizes was assessed using the Q statistic as well as Higgin's I ${ }^{2}$ statistic, which estimates the proportion of total variation in study estimates due to heterogeneity and ranges from 0 (no heterogeneity) to 1 (extreme heterogeneity). We further assessed whether the treatment effect varied between studies using paravertebral anesthesia alone vs paravertebral combined with GA when comparing with GA.

\section{Results}

One hundred eighty-seven PPA and 199 SOA randomized patients were included in the analysis. Patients were enrolled at the following sites: Cleveland Clinic Main Campus - Cleveland, Ohio, $n=53$; Mater Misericordiae University Hospital - Dublin, Ireland, $n=115$; Cleveland Clinic Fairview - Cleveland, Ohio, $n=16$; University of Louisville - Louisville, Kentucky, $n=11$; University of Vienna - Vienna, Austria, $n=106$; Cleveland Clinic Beachwood - Cleveland, Ohio, $n=49$; Cleveland Clinic Hillcrest - Cleveland, Ohio, $n=8$; Hospital Italiano Buenos Aires, Argentina, $n=7$; Düsseldorf University Hospital - Düsseldorf, Germany, $n=21$.

A CONSORT flow diagram is presented in Fig. 1. Morphometric and demographic characteristics of the patients in each treatment group were similar, as were the types of surgery (Table 1). Ninety-seven percent of outpatients assigned to the PPA group received the combination of paravertebral analgesia and GA. Among them, 63\% had tracheal intubation, and 34\% had laryngeal mask airways. Paravertebral catheters were inserted in $42 \%$
Table 1 Demographic and Morphometric Characteristics

\begin{tabular}{lcc}
\hline Factors & $\begin{array}{l}\text { Regional (PPA } \\
\text { group) }(n=187)\end{array}$ & $\begin{array}{l}\text { General-Opioid (SOA } \\
\text { group) }(n=199)^{\mathrm{b}}\end{array}$ \\
\hline Age $(\mathrm{yr})^{\mathrm{a}}$ & $58(11)$ & $55(12)$ \\
BMI $\left(\mathrm{kg} \cdot \mathrm{m}^{-2}\right)^{\mathrm{b}}$ & $28(8)$ & $28(7)$ \\
ASA status $^{\mathrm{c}}$ & $1.8(0.6)$ & $1.7(0.6)$ \\
I & $61(35 \%)$ & $66(35 \%)$ \\
II & $94(53 \%)$ & $106(56 \%)$ \\
III & $21(12 \%)$ & $16(9 \%)$ \\
Type of Surgery & & $29(15 \%)$ \\
Simple Mastectomy & $26(14 \%)$ & $23(12 \%)$ \\
Modified Radical & $19(11 \%)$ & $130(68 \%)$ \\
Wide Local Excision & $133(73 \%)$ & $9(5 \%)$ \\
$\quad$ w/ Node Dissection & & \\
Other & $3(2 \%)$ & \\
\hline
\end{tabular}

Data are reported as number (\%), means (SD)

PPA = paravertebral analgesia with propofol general anesthesia; $\mathrm{SOA}=$ sevoflurane general anesthesia combined with perioperative opioid analgesia; ASA = American Society of Anesthesiologists; $\mathrm{BMI}=$ body mass index

a, b, c, d indicate $6,2,11,6$ missing data points in the paravertebral group, and 4, 2, 11, 8 in the general-opioid group, respectively

of the PPA patients; none had a pneumothorax or spread of local anesthetic into the epidural space.

Both intention-to-treat and available data analyses included all $(n=386)$ patients and reached the same conclusions. Tests on all outcomes for non-inferiority were significant at the 0.025 level, as were the individual superiority tests. In one-tailed superiority tests (i.e., same direction as non-inferiority tests), the PPA group had significantly lower pain scores; reduced consumption of long-acting opioids, intraoperative fentanyl, and sevoflurane; and increased use of propofol when compared with the SOA group (all $P<0.001$ ) (Table 2
Fig. 1 CONSORT flow diagram

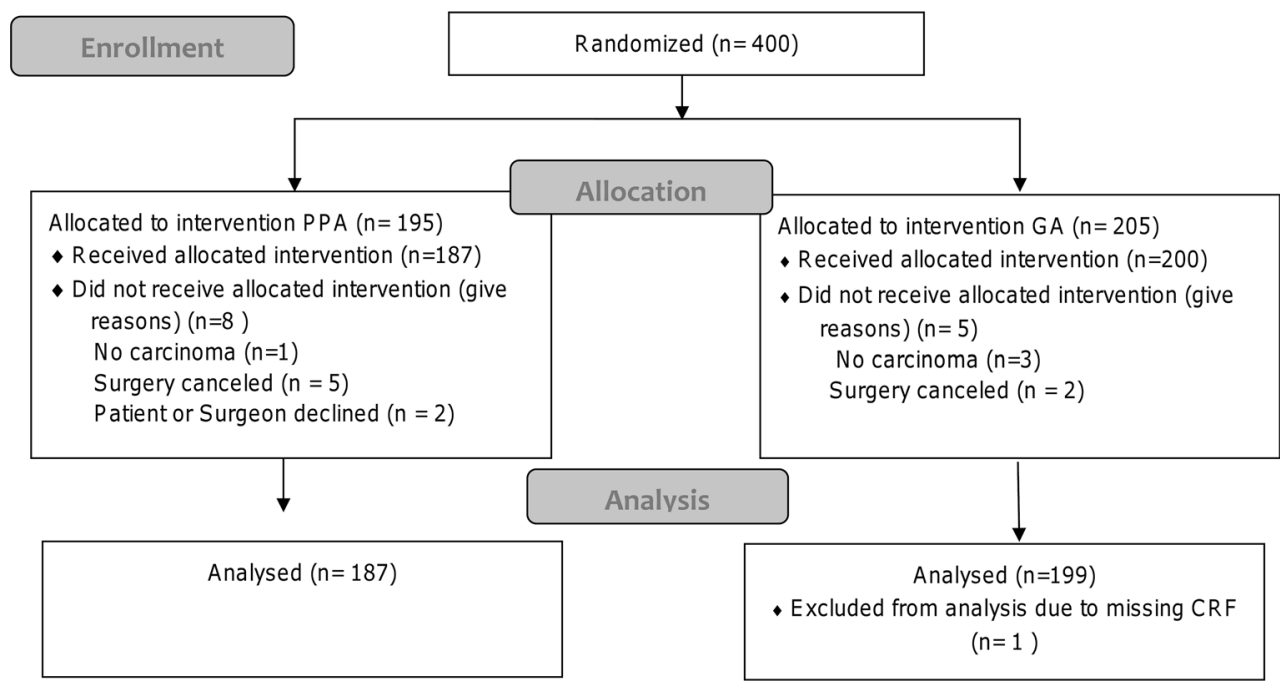




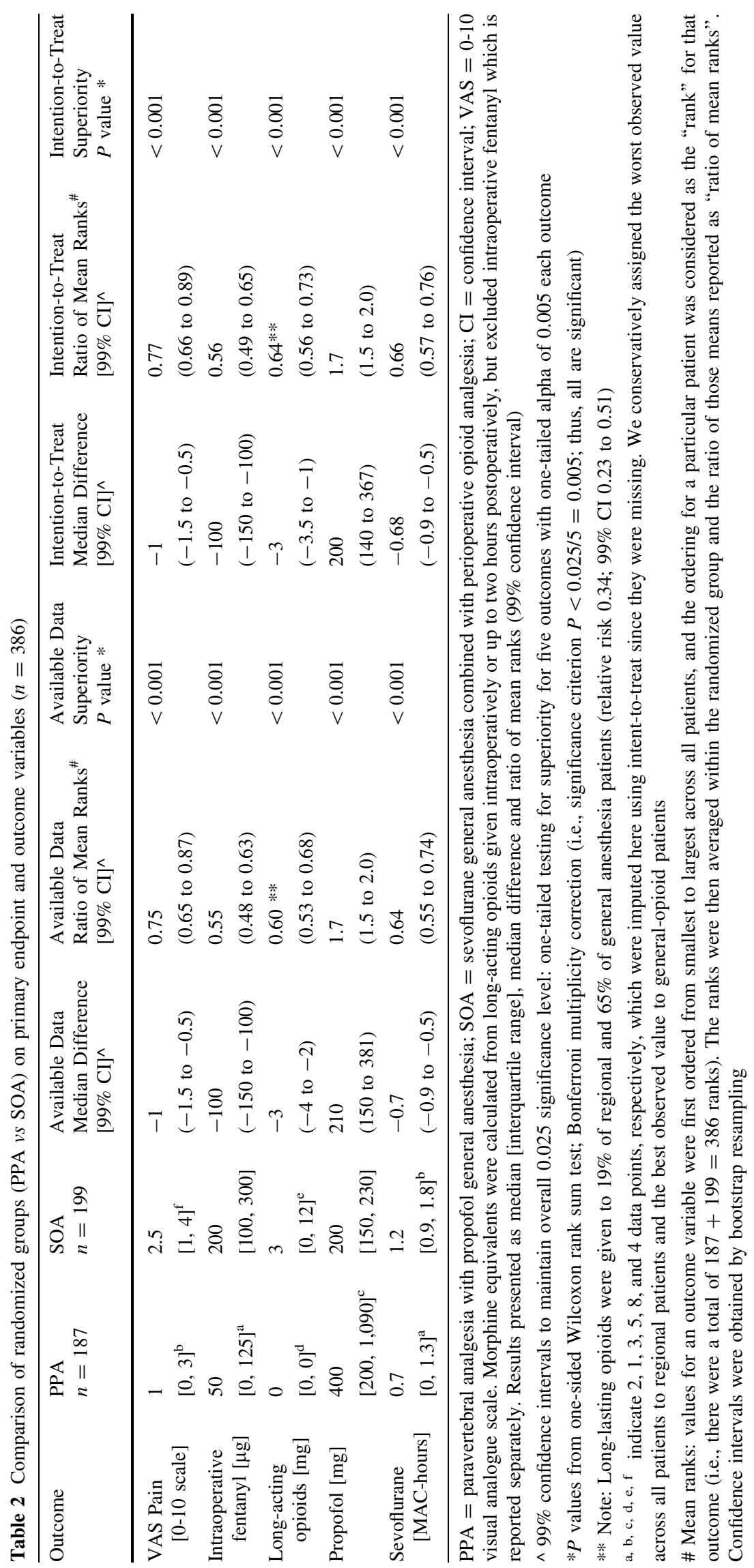


Fig. 2 Boxplots comparing randomized groups on each primary outcome using all available data. Box shows the interquartile range; horizontal line marks the median; whiskers extend to high and low values within 1.5 interquartile ranges of the box; circles are values beyond 1.5 interquartile ranges of the box; diamond shows the mean

Outcomes
VAS Pain Score
Long-acting opioids
Intraoperative fentany
Sevoflurane

Sevoflurane
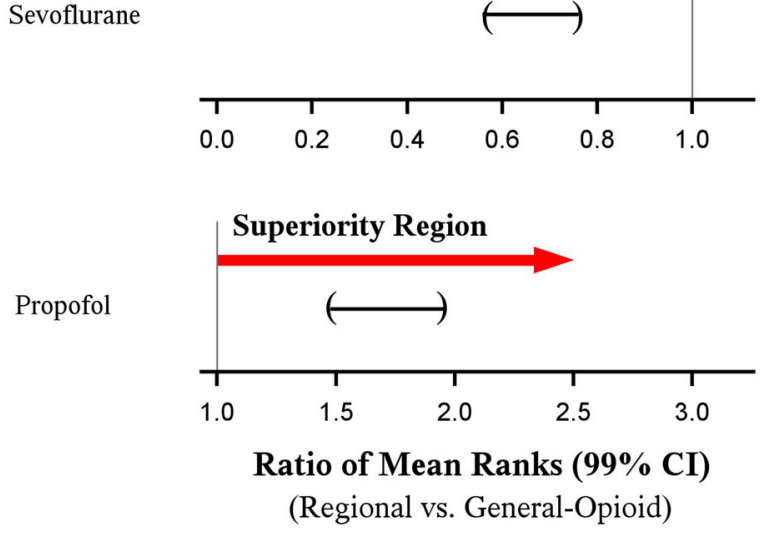

Fig. 3 Superiority plots showing the ratios of mean ranks (99\% confidence interval) between the regional and general groups in each primary outcome using intent-to-treat. Parentheses indicate two-sided 99\% confidence intervals estimated using bootstrap resampling. Joint hypothesis testing of the five primary outcomes indicated effectiveness of paravertebral analgesia over general anesthesia because all five confidence intervals fall into the superiority regions: less pain, less use of intraoperative fentanyl, less use of long-acting opioids, less use of volatile anesthetics, and increased use of propofol
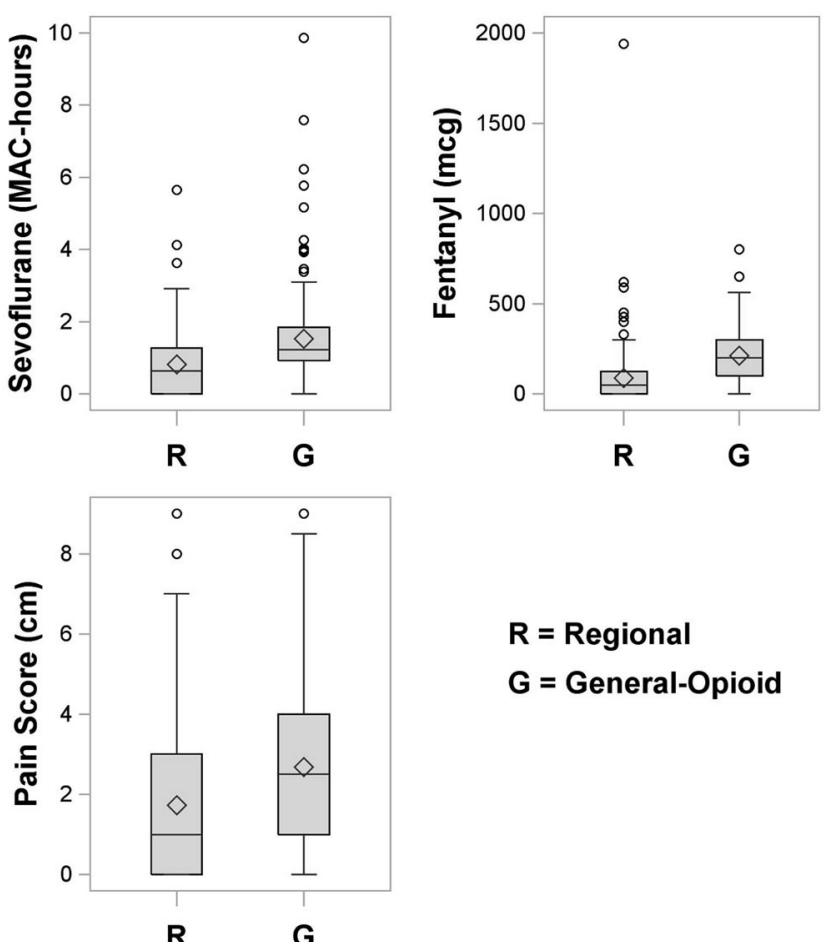

$$
\begin{aligned}
& \mathbf{R}=\text { Regional } \\
& \mathbf{G}=\text { General-Opioid }
\end{aligned}
$$

and Fig. 2). Long-lasting opioids were given to $19 \%$ of regional patients and $65 \%$ of GA patients (relative risk, 0.34; $99 \%$ CI: 0.23 to 0.51 ).

Extending beyond the first two postoperative hours, we found less opioid use in PPA vs SOA anesthesia on postoperative day 1 (17\% of patients vs $33 \%$ of patients, respectively; $P<0.001$ for any use and amount of opioids; relative risk $0.59 ; 99 \%$ CI: 0.49 to 0.75 ), but no difference on postoperative day 2 for any use (6\% vs 10\%, respectively; relative risk 0.91 ; $99 \%$ CI: 0.92 to 1.11 ; $P=0.13)$. The data in this study were analyzed by intention-to-treat analysis. Where patients were allocated to a PPA technique, but where the paravertebral block failed (as can happen in approximately $10 \%$ of cases), ${ }^{4}$ anesthesiologists would default to a "balanced GA", typically involving sevoflurane as in the SOA arm of the study. Therefore, when the data were analyzed, a small number of patients randomized to receive propofol only actually received sevoflurane, rendering the mean sevoflurane value above zero in the PPA group.

Results are displayed as median difference between the groups and $99 \% \mathrm{CI}$ as well as the estimated ratio of mean ranks and 99\% CI. For example, in the intent-to-treat analysis, the estimated ratio (99\% CI) of sevoflurane mean ranks of 0.66 (0.57 to 0.76$)$ estimates that regional anesthesia reduced the average use of sevoflurane (on the rank scale instead of actual scale) by $34 \%$ (99\% CI 24 to 43). Similarly, in the available data analysis, regional 


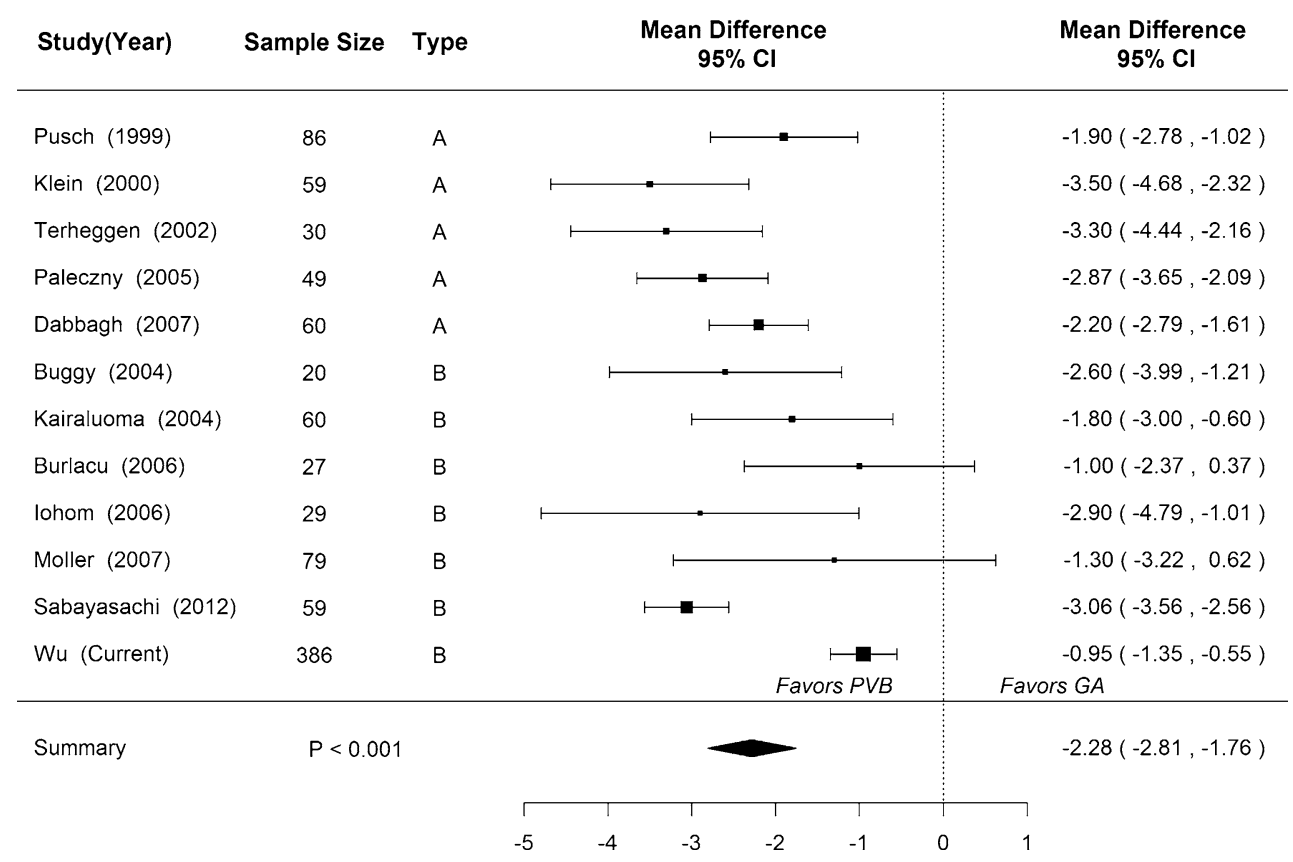

Fig. 4 Meta-analysis comparing paravertebral analgesia (with or without concomitant general anesthesia) vs general anesthesia on worst pain score in the immediate two postoperative hours. Treatment effect expressed as mean difference in worst pain score (10-cm visual analogue scale or $0-10$ numerical rating scale). Type A studies compared paravertebral alone vs general anesthesia while Type B compared paravertebral combined with general anesthesia $v s$ general anesthesia. Overall, paravertebral analgesia (with or without general anesthesia) reduced pain over general anesthesia by an estimated 2.28 (95\% confidence interval: 1.76 to $2.81 ; P<0.001$ ). Heterogeneity was observed (Q statistic $P<0.001$ and $\mathrm{I}^{2}=76 \%$ ), but all point estimates of the treatment effect favoured paravertebral blocks

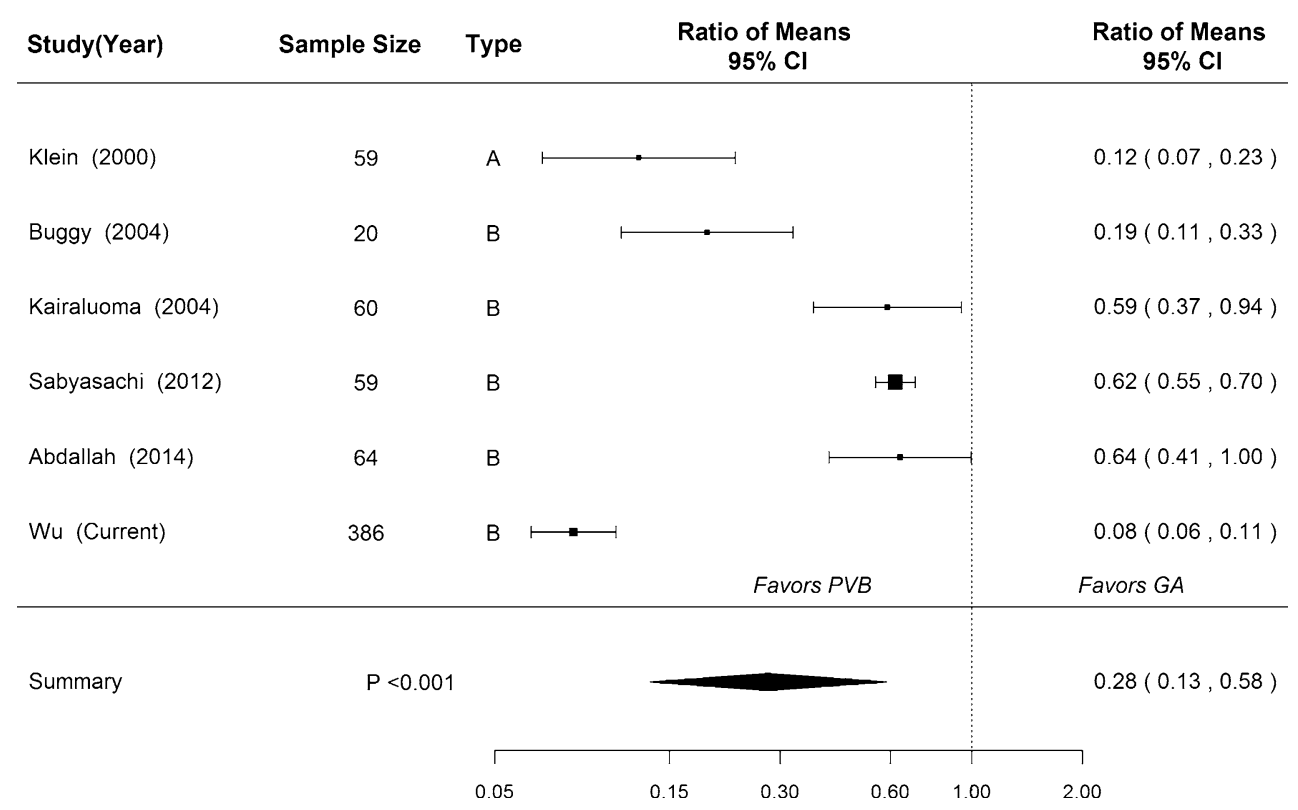

Fig. 5 Meta-analysis comparing paravertebral analgesia (with or without concomitant general anesthesia) vs general anesthesia on postoperative use of analgesics (morphine $\mathrm{mg}$ ) in the immediate two postoperative hours. Treatment effect expressed as ratio of geometric means of opioid consumption. Type A studies compared paravertebral anesthesia alone vs general anesthesia, while Type B compared paravertebral combined with general anesthesia $v s$ general anesthesia.
Overall, paravertebral (or paravertebral combined with general anesthesia) reduced opioid consumption by an estimated $72 \%(95 \%$ confidence interval: 42 to 87 ), with mean ratio of 0.28 (95\% confidence interval: 0.13 to 0.58$), P<0.001$. Heterogeneity was observed (Q statistic $P<0.001$ and $\mathrm{I}^{2}=97 \%$ ), but all point estimates of the treatment effect favoured paravertebral blocks 
anesthesia reduced the amount of sevoflurane used by an estimated $36 \%$ (99\% CI $26 \%$ to $45 \%$ ).

Joint hypothesis testing of the primary outcomes indicated effectiveness of PPA over SOA because superiority in the pre-specified direction was observed for all outcomes (i.e., all superiority tests $P<0.001$ and all estimated 99\% CIs fell into the superiority regions) (Fig. 3).

The results of our meta-analysis are outlined in Figs. 4 and 5. For worst pain score in the immediate two postoperative hours, the recent relevant meta-analysis ${ }^{23}$ contained five eligible trials ${ }^{24-28}$ evaluating paravertebral anesthesia as a single technique $v s$ GA and five eligible trials $^{14,15,29-31}$ evaluating the combination of paravertebral analgesia and GA vs GA alone. One additional recent trial $^{32}$ was identified and included in our meta-analysis along with our current results.

For actual opioid consumption in the immediate two postoperative hours, the recent relevant meta-analysis ${ }^{4}$ contained one eligible trial evaluating paravertebral anesthesia as a single technique $v s \mathrm{GA}^{25}$ and two others evaluating the combination of paravertebral analgesia and GA vs GA alone. ${ }^{15,29}$ Two additional recent trials ${ }^{32,33}$ were identified, which were included in our meta-analysis along with our current result.

For worst pain score in the immediate two postoperative hours, our meta-analysis of 12 studies showed that paravertebral analgesia (with or without concomitant GA) reduced the worst pain score, on a 0-10 scale, by an estimated 2.3 points (95\% CI: 1.8 to 2.8 ) across studies (Fig. 4). Likewise, for opioid consumption in the immediate two postoperative hours, our meta-analysis of six studies showed that paravertebral analgesia (with or without concomitant GA) reduced analgesic use after surgery by an estimated $72 \%$ (95\% CI: 42 to 87 ; $P<0.001)$ (Fig. 5).

Significant heterogeneity was found across studies using either the Q statistic (both $P<0.001$ ) or $\mathrm{I}^{2}$, with $\mathrm{I}^{2}$ of $76 \%$ (95\% CI: 48 to 92) for worst pain score and 97\% (95\% CI: 92 to 99) for morphine consumption. Nevertheless, estimated treatment effects for all studies were in the same direction, always favouring paravertebral blocks. There was little evidence of publication bias in the funnel plots (not shown). There was also no difference in the treatment effects for paravertebral alone $v s$ GA compared with combined paravertebral analgesia and GA vs GA alone (interaction $P=0.14$ for pain score and $P=0.063$ for opioid consumption).

\section{Discussion}

Only 11 published trials with only 558 patients in total evaluated worst pain in the immediate two postoperative hours in patients randomized to paravertebral analgesia (with or without concomitant GA) vs GA alone. ${ }^{14,15,24-32}$ Only five published trials ${ }^{15,25,29,32,33}$ with only 262 patients in total evaluated actual opioid consumption in the immediate two postoperative hours in patients randomized to paravertebral analgesia (with or without concomitant GA) vs GA alone. Our results are generally consistent with previous reports and show that patients given paravertebral analgesia had less immediate postoperative pain and required fewer morphine sulphate equivalents.

It is not surprising that paravertebral blocks are analgesic and reduce opioid use; however, the largest previous study on immediate postoperative pain included only 86 patients ${ }^{14,24}$ and the largest study on opioid sparing included only 64 patients. ${ }^{24}$ Furthermore, all previous studies were single-centre. Therefore, considerable uncertainty remained over the magnitude of the paravertebral effect and the extent to which it was generalizable. Our study of 386 patients is thus a substantial contribution to available literature, and furthermore, it includes patients from nine international centres. We thus provide a robust estimate of treatment effect that was not previously available.

Although these results, when added to the pain and opioid reduction meta-analyses, indicate effective pain control from paravertebral blockade in the breast cancer population, the original purpose of our overall investigation was to reduce the risk of cancer recurrence (as well as chronic pain). Accordingly, in this study we are essentially comparing two distinct "bundles" of anesthetic technique, one which putatively might help resist cancer and the other being "standard" care. As intended, we have shown that they have effectively produced two distinct groups.

Analgesia was satisfactory in both of our study groups. Clinically, optimal postoperative analgesia might be characterized by a VAS score $<4$. To achieve this clinical goal in our study protocol, intravenous morphine sulphate was titrated to a respiratory rate of 12-14 breaths $\cdot \mathrm{min}^{-1}$ near the end of surgery. In our PPA group, the surgeon administered injections of $0.5 \%$ ropivacaine to block cervical and contralateral thoracic nerves that also contribute to the innervation of the breast. Postoperatively, analgesia was primarily provided by a regional block and was supplemented with acetaminophen and/or nonsteroidal anti-inflammatory drugs. Supplemental morphine or a similar long-acting opioid was provided if pain relief was inadequate. In our SOA group, however, morphine or a similar long-acting opioid was provided postoperatively as needed, either intravenously or via patient-controlled pump.

Long-lasting opioids were given to $19 \%$ of PPA and $65 \%$ of SOA patients. It is thus unsurprising that 
paravertebral analgesia provides better analgesia, and this is generally consistent with previous findings. Nonetheless, patients assigned to SOA averaged about 2.5 on a VAS scale, while mean pain scores were only about 1 in those given PPA analgesia. Only three previous studies evaluated use of volatile anesthetics, and the results were inconsistent. $^{15}$

As might be expected, previous small studies generally overestimated treatment effect, with some reporting differences exceeding three points on an 11-point scale. ${ }^{24}$ Combining all studies, the overall pain benefit was a reduction by more than 2 , an amount which was highly statistically significant but probably also reflects a clinically important reduction in discomfort.

Our meta-analysis, which included five previous trials $^{15,25,29,32,33}$ and our current patients, showed that paravertebral analgesia reduced opioid consumption by an estimated $72 \%$ (95\% CI: 48 to $87 ; P<0.001$ ). There was heterogeneity, but once again, point estimates all identified benefit. The overall benefit was an estimated reduction by a factor of five $(72 \%)$ in opioid use, a reduction that is of obvious clinical benefit as well as being statistically significant. Our current results, along with previous studies, thus show that paravertebral analgesia is effective in reducing both postoperative pain and the need for opioids and volatile anesthetics.

We caution that we assumed a log-normal distribution for the opioid data in each of the six studies in the metaanalysis, since opioid consumption is often log-normal in practice. Nevertheless, this leads to some uncertainty in the results of the meta-analysis since we did not have the raw data to verify the assumption. For example, from the raw data in our current study, we estimated a $36 \%$ reduction in mean ranks of opioid consumption, which is conservative because it is on the rank scale. Assuming instead a lognormal distribution and inserting the observed mean and standard deviation in the meta-analysis, we attain a much larger $(92 \%)$ reduction, which is likely an overestimate. The true value presumably lies between the two estimates.

We wanted the data to be valid and applicable to a wide range of practice, and we thought that creating two excessively unusual groups might make any differences seem remote or unlikely to be achieved in everyday practice. Therefore, our conclusions represent real-world consequences of paravertebral analgesia rather than the relatively predictable results of a highly specified protocol.

We used a joint hypothesis testing methodology for the endpoints and outcomes such that we claimed superiority of PPA over SOA only if it was superior on at least one of the outcomes and non-inferior on the others. ${ }^{20}$ With this joint testing of the outcomes, we avoided the confusion of claiming, for example, less opioid consumption for regional anesthesia but more pain as well. We were also able to avoid adjustments to the significance criterion in assessing non-inferiority for each outcome because a significant conclusion of non-inferiority was required for all outcomes in order to test for superiority. For superiority testing, however, we appropriately applied a multiple comparison procedure to control the type I error; superiority was not required on all outcomes (only one or more) to claim greater efficacy of regional anesthesia vs GA.

Our results should be interpreted with a degree of caution. We have shown only that the PPA technique altered the drug use and analgesic variables as it was envisaged to do in this trial. The reduction in opioid consumption and VAS scores seen by others has indeed been confirmed, albeit with larger numbers.

In summary, our large multicentre trial nearly doubles the total number of breast cancer surgery patients (in the available literature) randomized to paravertebral analgesia (with propofol GA) vs sevoflurane GA with opioid analgesia. We have shown that, in the immediate two postoperative hours, a paravertebral analgesia-based anesthetic reduces pain scores and opioid requirements and also reduces the doses of volatile anesthetics. Our meta-analysis concluded that pain is reduced by about two points (on a 0-10 VAS scale) and that opioid use is reduced by about $70 \%$ in the immediate postoperative two hours.

Funding This work was supported by the Austrian National Bank Fund (Vienna, Austria) and the Sisk Healthcare Foundation (Mater Misericordiae Hospital Dublin, Ireland).

Conflicts of interest Donal Buggy's institution received a grant from Sisk Healthcare. Edith Fleischmann's institution received a grant from the Austrian National Bank. None of the authors has any personal financial interest in this research.

\section{Appendix}

Dates of Research Ethics Committee (Institutional Ethics Review Board) approval:

Mater Misericordiae University Hospital Dublin Ireland: August 2008;

Cleveland Clinic, OH, USA: October 2008;

University of Vienna, Austria: February 2009;

University of Dusseldorf: September 2010.

\section{References}

1. Exadaktylos AK, Buggy DJ, Moriarty DC, Mascha E, Sessler DI. Can anesthetic technique for primary breast cancer surgery affect recurrence or metastasis? Anesthesiology 2006; 105: 660-4. 
2. Karmakar MK. Thoracic paravertebral block. Anesthesiology 2001; 95: 771-80.

3. Tahiri $Y$, de Tran $Q H$, Bouteaud J, et al. General anaesthesia versus thoracic paravertebral block for breast surgery: a metaanalysis. J Plast Recontr Aesthet Surg 2011; 64: 1261-9.

4. Schnabel A, Reichl SU, Kranke P, Pogatzki-Zahn EM, Zahn PK. Efficacy and safety of paravertebral blocks in breast surgery: a meta-analysis of randomized controlled trials. Br J Anaesth 2010; 105: 842-52.

5. Procopio MA, Rassias AJ, DeLeo JA, Pahl J, Hildebrandt L, Yeager MP. The in vivo effects of general and epidural anesthesia on human immune function. Anesth Analg 2001; 93: 460-5.

6. O'Donnell NG, McSharry CP, Wilkinson PC, Asbury AJ. Comparison of the inhibitory effect of propofol, thiopentone and midazolam on neutrophil polarization in vitro in the presence or absence of human serum albumin. Br J Anaesth 1992; 69: 70-4.

7. Melamed R, Bar-Yosef S, Shakhar G, Shakhar K, Ben-Eliyahu S. Suppression of natural killer cell activity and promotion of tumor metastasis by ketamine, thiopental, and halothane, but not by propofol: mediating mechanisms and prophylactic measures. Anesth Analg 2003; 97: 1331-9.

8. Mammoto T, Mukai M, Mammoto A, et al. Intravenous anesthetic, propofol inhibits invasion of cancer cells. Cancer Lett 2002; 184 : 165-70.

9. Sacerdote P, Bianchi M, Gaspani L, et al. The effects of tramadol and morphine on immune responses and pain after surgery in cancer patients. Anesth Analg 2000; 90: 1411-4.

10. Beilin B, Shavit $Y$, Hart J, et al. Effects of anesthesia based on large versus small doses of fentanyl on natural killer cell cytotoxicity in the perioperative period. Anesth Analg 1996; 82: 492-7.

11. Gupta K, Kshirsagar S, Chang L, et al. Morphine stimulates angiogenesis by activating proangiogenic and survival-promoting signaling and promotes breast tumor growth. Cancer Res 2002; 62: 4491-8.

12. Page GG, Blakely WP, Ben-Eliyahu $S$. Evidence that postoperative pain is a mediator of the tumor-promoting effects of surgery in rats. Pain 2001; 90: 191-9.

13. Sessler DI, Ben-Eliyahu S, Mascha EJ, Parat MO, Buggy DJ. Can regional analgesia reduce the risk of recurrence after breast cancer? Methodology of a multicenter randomized trial. Contemp Clin Trials 2008; 29: 517-26.

14. Moller JF, Nikolajsen L, Rodt SA, Ronning H, Carlsson PS. Thoracic paravertebral block for breast cancer surgery: a randomized double-blind study. Anesth Analg 2007; 105: 1848-51.

15. Kairaluoma PM, Bachmann MS, Korpinen AK, Rosenberg PH, Pere PJ. Single-injection paravertebral block before general anesthesia enhances analgesia after breast cancer surgery with and without associated lymph node biopsy. Anesth Analg 2004; 99: 1837-43.

16. Ashburn MA, Lipman AG, Carr D, Rubingh C. Principles of Analgesic use in the Treatment of Acute Pain and Cancer Pain. 5th ed. Glenview, IL: American Pain Society; 2003.
17. Gibb DB, Pikler N. Piritramide-a new long-acting analgesic. Anaesth Intensive Care 1973; 1: 308-14.

18. Kay B. A clinical investigation of piritramide in the treatment of postoperative pain. Br J Anaesth 1971; 43: 1167-71.

19. Myles PS, Troedel S, Boquest M, Reeves $M$. The pain visual analog scale: is it linear or nonlinear? Anesth Analg 1999; 89: 1517-20.

20. Mascha EJ, Turan A. Joint hypothesis testing and gatekeeping procedures for studies with multiple endpoints. Anesth Analg 2012; 114: 1304-17.

21. Rohmel $J$, Gerlinger $C$, Benda $N$, Lauter $J$. On testing simultaneously non-inferiority in two multiple primary endpoints and superiority in at least one of them. Biom J 2006; 48: 916-33.

22. Berger RL. Multiparameter hypothesis testing and acceptance sampling. Technometrics 1982; 24: 295-300.

23. Efron B, Tibshirani RJ. An Introduction to the Bootstrap. New York: Chapman and Hall; 1993.

24. Pusch F, Freitag H, Weinstabl C, Obwegeser R, Huber E, Wildling $E$. Single-injection paravertebral block compared to general anaesthesia in breast surgery. Acta Anaesthesiol Scand 1999; 43: 770-4.

25. Klein SM, Bergh A, Steele SM, Georgiade GS, Greengrass RA. Thoracic paravertebral block for breast surgery. Anesth Analg 2000; 90: 1402-5.

26. Terheggen MA, Wille F, Borel Rinkes IH, Ionescu TI, Knape JT. Paravertebral blockade for minor breast surgery. Anesth Analg 2002; 94: 355-9.

27. Dabbagh A, Elyasi $H$. The role of paravertebral block in decreasing postoperative pain in elective breast surgeries. Med Sci Monit 2007; 13: CR464-7.

28. Paleczny J, Loniewska-Paleczny E, Pysz M, Hura G. Thoracic paravertebral block versus general anaesthesia in breast surgery (Polish). Anestezjologia Intensywna Terapia 2005; 37: 12-6.

29. Buggy DJ, Kerin MJ. Paravertebral analgesia with levobupivacaine increases postoperative flap tissue oxygen tension after immediate latissimus dorsi breast reconstruction compared with intravenous opioid analgesia. Anesthesiology 2004; 100: 375-80.

30. Burlacu CL, Frizelle HP, Moriarty DC, Buggy DJ. Fentanyl and clonidine as adjunctive analgesics with levobupivacaine in paravertebral analgesia for breast surgery. Anaesthesia 2006; 61: 932-7.

31. Iohom $G$, Abdalla H, O'Brien $J$, et al. The associations between severity of early postoperative pain, chronic postsurgical pain and plasma concentration of stable nitric oxide products after breast surgery. Anesth Analg 2006; 103: 995-1000.

32. Das S, Bhattacharya P, Mandal MC, Mukhopadhyay S, Basu SR, Mandol BK. Multiple-injection thoracic paravertebral block as an alternative to general anaesthesia for elective breast surgeries: A randomised controlled trial. Indian J Anaesth 2012; 56: 27-33.

33. Abdallah FW, Morgan PJ, Cil T, et al. Ultrasound-guided multilevel paravertebral blocks and total intravenous anesthesia improve the quality of recovery after ambulatory breast tumor resection. Anesthesiology 2014; 120: 703-13. 